\title{
Prevention of bile spillage during cholecystectomy: current evidences and considerations
}

\author{
Maurizio Zizzo ${ }^{1,2}$, Lorenzo Manzini ${ }^{1}$, Magda Zanelli ${ }^{3}$, Loredana De Marco ${ }^{3}$, Carolina Castro Ruiz ${ }^{4}$, \\ Antonio Manenti ${ }^{5}$, Valerio Annessi ${ }^{4}$ \\ ${ }^{1}$ Surgical Oncology Unit, Azienda Unità Sanitaria Locale-IRCCS di Reggio Emilia, Arcispedale Santa Maria Nuova di Reggio Emilia, Reggio Emilia, \\ Italy; ${ }^{2}$ Clinical and Experimental Medicine PhD Program, University of Modena and Reggio Emilia, Modena, Italy; ${ }^{3}$ Pathology Unit, Azienda Unità \\ Sanitaria Locale-IRCCS di Reggio Emilia, Arcispedale Santa Maria Nuova di Reggio Emilia, Reggio Emilia, Italy; ${ }^{4}$ General Surgery Unit, Azienda \\ Unità Sanitaria Locale-IRCCS di Reggio Emilia, Ospedale Civile di Guastalla, Reggio Emilia, Italy; ${ }^{5}$ Department of General Surgery, Azienda \\ Ospedaliero-Universitaria - Policlinico, Modena, Italy \\ Correspondence to: Maurizio Zizzo. Surgical Oncology Unit, Azienda Unità Sanitaria Locale-IRCCS di Reggio Emilia, Arcispedale Santa Maria Nuova \\ di Reggio Emilia, Viale Risorgimento, 80, 42123 Reggio Emilia, Italy. Email: zizzomaurizio@gmail.com.
}

Submitted Oct 16, 2019. Accepted for publication Oct 31, 2019.

doi: $10.21037 /$ hbsn.2019.11.25

View this article at: http://dx.doi.org/10.21037/hbsn.2019.11.25

We really appreciated Geramizadeh, "Bile spillage and incidental gall bladder adenocarcinoma", and Sandstrom et al., "Bile spillage should be avoided in elective cholecystectomy" $(1,2)$. Both papers have been recently published in HepatoBiliary Surgery and Nutrition, 2019 (1,2). Starting from an analysis of the recent paper by Horkoff et al. "Adverse outcomes after bile spillage in incidental gall bladder cancer" in Annals of Surgery April 2019, they introduced a comprehensive discussion about epidemiology, diagnosis, therapy, prognostic factors and outcomes of incidental gallbladder cancer (1-3). They analyzed the possible risks related to bile spillage after cholecystectomy, in particular $(1,2)$.

Incidental gallbladder cancers have been reported in 0.2 $3 \%$ cholecystectomies (1). Approximately $30 \%$ gallbladder cancers are accidentally diagnosed on surgical specimen after cholecystectomy for presumed benign disease (3). However, most gallbladder cancers present an advanced disease and preclude curative surgery (1,3-5). Indeed, only $10 \%$ incidentally diagnosed gallbladder cancers are resectable $(1,3)$.

During index cholecystectomy, which is generally performed in non-neoplastic diseases, such as gallstones or acute cholecystitis, intentional decompression, accidental gallbladder perforation, incomplete closing of cystic duct, performance of intraoperative cholangiography may lead to spillage of bile in up $26.9 \%$ cholecystectomies, thus leading to dissemination of neoplastic cells in about $0.5 \%$ cholecystectomies (1-3). Invasion depth, extent of resection and bile spillage represent important prognostic factors in gallbladder cancer $(1-3,6)$.

Horkoff et al. retrospectively analyzed a population of 82 patients who underwent laparoscopic cholecystectomy that incidentally diagnosed gallbladder adenocarcinoma on postoperative histological examination (T1b stage or more) (3). If compared to patients without bile spillage, patients with bile spillage ( $67 \%$ of the entire population) recorded significantly higher rate of carcinomatosis ( $24 \%$ vs. $4 \%$, odds 8.04), greater distance spread rate $(72 \%$ vs. $37 \%)$ but smaller locoregional spread rate (10\% vs. 26\%) (3). Furthermore, median disease-free survival (DFS) and median overall survival (OS) were worse in patients with bile spillage than in patients without bile spillage (7.76 months vs. 22.33 ones; 15.83 months vs. 31.27 ones) (3).

While bile spillage was the only factor which independently predicted a reduced tendency to proceed with re-resection, bile spillage in addition to advanced age independently predicted failure to achieve $\mathrm{R} 0$ re-resection margins (3). If compared to patients who had undergone radical re-resection, patients, who had failed radical reresection, recorded worse values: median OS $15.2 \mathrm{vs}$. 40.1 months; median DFS 7.7 vs. 21.9 months (3).

Recently published data by Blakely et al. seem to be in line with the above results (7). Seventy-nine patients affected by pathologically-confirmed gallbladder cancer 
(T1b stage or more) were retrospectively analyzed (7). Out of them, 41 underwent cholecystectomy, 16 had radical cholecystectomy, 9 received cholecystectomy plus Rouxen-Y hepaticojejunostomy (7). When compared to the 52 patients without bile spillage, 14 patients in the bile spillage group recorded a significantly higher local progression rate $(57.1 \%$ vs. $25 \%)$ and a unsignificantly higher peritoneal progression rate $(42.9 \%$ vs. $21.2 \%)$ (7). At multivariate analysis, bile spillage was one independent prognostic factor for reduced DFS (7). Deeper analysis of just subpopulation undergoing index cholecystectomy (41 patients) showed association of bile spillage (12 patients) with significant median DFS reduction (5.7 vs. 22.2 months) and a nonsignificant OS reduction (7).

In the light of scientific data, experience at our tertiary care center and results by other Italian Healthcare Institution, we would like to introduce our considerations on laparoscopic cholecystectomy, which represents the gold standard and the most frequently adopted method of gallbladder resection (1).

* When faced with an "easy" cholecystectomy, some surgeons perform direct extraction of surgical specimen through the port site, rather than making use of endobag. Given the above-mentioned results, we would suggest endobag for all laparoscopic cholecystectomies, regardless of the ease of surgical procedure. Nevertheless, current data offered by literature on the risks of carcinomatosis or recurrences do not show significantly different outcomes when using endobag and not using it (8).

* Following dissection of Calot's triangle, isolation with section of cystic structures and detachment of gallbladder from liver, some surgeons are prone to "leave" surgical specimen inside abdominal cavity, and immediately engage in hemostasis and/or placement of drainage. We suggest they introduce surgical specimen into the endobag, as soon as its detachment is completed.

* Generally, surgical report describes steps of surgical procedure with no mention of any bile spillage. However, given the extremely serious consequences of bile spillage in patients affected by incidental gallbladder cancer and in accordance with Blakely et al. (7) we suggest intraoperative bile spillage be essential integral part of the operative note.

- In addition to laparoscopic appendectomy, laparoscopic cholecystectomy, is one of the first most frequently performed interventions by young surgeons during their learning curve in minimally invasive surgery. In our mind, they should be immediately taught how to pay attention both to procedure-related possible bilio-vascular damage and to increased risk of bile spillage.

* Conversely, being well aware of their advanced surgical technical skills, more experienced surgeons may have an "easy" approach to laparoscopic cholecystectomy, thus "easing" bile spillage. We remind them to keep in mind such risk although it is minimal.

\section{Acknowledgments}

We thank Dr. Daniela Masi (AUSL-IRCCS di Reggio Emilia) for support in English editing.

\section{Footnote}

Conflicts of Interest: The authors have no conflicts of interest to declare.

Ethical Statement: The authors are accountable for all aspects of the work in ensuring that questions related to the accuracy or integrity of any part of the work are appropriately investigated and resolved. Local ethics committee (Comitato Etico dell'Area Vasta Emilia Nord, Sede presso la AUSL - IRCCS di Reggio Emilia, Edificio Spallanzani, Viale Umberto I n. $50,1^{\circ}$ piano - Infrastruttura Ricerca e Statistica, Italy) ruled out any need for formal ethics approval.

\section{References}

1. Geramizadeh B. Bile spillage and incidental gall bladder adenocarcinoma. HepatoBiliary Surg Nutr 2019;8:646-8.

2. Sandstrom P, Bjornsson B. Bile spillage should be avoided in elective cholecystectomy. HepatoBiliary Surg Nutr 2019;8:640-2.

3. Horkoff MJ, Ahmed Z, Xu Y, et al. Adverse Outcomes After Bile Spillage in Incidental Gallbladder Cancers: A Population-based Study. Ann Surg 2019. [Epub ahead of print].

4. Rawla P, Sunkara T, Thandra KC, et al. Epidemiology of gallbladder cancer. Clin Exp Hepatol 2019;5:93-102.

5. Cherkassky L, D'Angelica M. Gallbladder Cancer: Managing the Incidental Diagnosis. Surg Oncol Clin N Am 2019;28:619-30. 
6. de Aretxabala X. Biliary spillage a new prognostic factor in gallbladder cancer? HepatoBiliary Surg Nutr 2019;8:537-8.

7. Blakely AM, Wong $\mathrm{P}$, Chu $\mathrm{P}$, et al. Intraoperative bile spillage is associated with worse survival in gallbladder

Cite this article as: Zizzo M, Manzini L, Zanelli M, De Marco L, Castro Ruiz C, Manenti A, Annessi V. Prevention of bile spillage during cholecystectomy: current evidences and considerations. Hepatobiliary Surg Nutr 2020;9(1):109-111. doi: 10.21037/hbsn.2019.11.25 adenocarcinoma. J Surg Oncol 2019;120:603-10.

8. Søreide K, Guest RV, Harrison EM, et al. Systematic review of management of incidental gallbladder cancer after cholecystectomy. Br J Surg 2019;106:32-45. 